\title{
Clarification des lactosérums doux par agrégation thermocalcique de la matière grasse résiduelle
}

\author{
J. FAUQUANT, Estella VIECO, G. BRULE \\ et J.-L. MAUBOIS
}

\section{Ré s u m é}

La clarification du lactosérum doux de fromagerie a été étudiée en mettant à profit la propriété des lipoprotéines de s'agréger en présence de calcium ionisé sous l'effet d'un traitement thermique. L'examen systématique de la quantité de calcium ajoutée au lactosérum, du $\mathrm{pH}$ à maintenir, dans le mélange et de la température de thermisation (le temps de chambrage étant fixé à $20 \mathrm{~s}$ ) a permis d'optimiser le procédé proposé c'est-à-dire l'obtention d'un lactosérum ayant une limpidité voisine de celle de l'ultrafiltrat de lait avec le minimum de pertes en protéines dans le sédiment. Ces pertes étaient limitées à environ $15 \%$ des matières azotées initiales dans les conditions retenues, à savoir : ajout de 0,8 g.l l-1 $^{-1}$ de calcium (sous forme de $\mathrm{CaCl}_{2}$ ), $\mathrm{pH}$ maintenu à 6,0 et température de traitement fixée à $78-79^{\circ} \mathrm{C}$.

L'agrégation et la réduction d'hydrophilie des complexes lipoprotéiques sous l'effet du traitement proposé entraîne leur décantation rapide à basse température. L'analyse des surnageants et des sédiments a permis de montrer que $80 \%$ de la $\beta$-lactoglobuline et $85 \%$ de l'o-lactalbumine initialement présentes dans le lactosérum, restaient solubles dans le produit clarifié.

Le lactosérum clarifié constitue une matière première idéale pour la préparation de boissons aromatisées ou pour la préparation d'une base pâtissière à pouvoir moussant très élevé. Du fait de sa composition et de ses propriétés fonctionnelles, notamment émulsifiantes, le sédiment devrait, aussi, quant à lui trouver de nom-

Laboratoire de Recherches de Technologie Laitière (I.N.R.A.), 65, rue de SaintBrieuc - 35042 Rennes cedex. 
breux débouchés aussi bien dans les produits laitiers classiques qu'en alimentation diététique.

Mots clés

Clarification - Lactosérum - Interactions - Calcium - Lipoprotéeines.

\section{S u m m a ry}

CLARIFICATION OF SWEET CHEESE WHEY BY THERMOCALCIC AGGREGATION OF RESIDUAL FAT

Clarification of sweet cheese whey was studied by using the property of lipoproteins to aggregate under heat treatment when ionic calcium is added. Optimization of process parameters was obtained through systematical study of amount of calcium to be added to the whey, $\mathrm{pH}$ adjustment and heating temperature, holding time being fixed at $20 \mathrm{~s}$. A clarified whey with an optical density at $600 \mathrm{~nm}$ similar to the one of milk ultrafiltrate and with a nitrogenous matter content representing $85 \%$ of those existing in the original whey was obtained when 8. g.t-1 of calcium as $\mathrm{CaCl}_{2}$ was added, when $\mathrm{pH}$ was adjusted to 6.0 and when heating temperature was $78-79^{\circ} \mathrm{C}$.

The proposed whey treatment leads to an aggregation and a reduction of hydrophily of lipoproteic complexes which are quickly decanting at low temperature. Supernatant and sediment analysis showed that around $80 \%$ of original B-lactoglobulin and $85 \%$ of initial ö-lactalbumine remain soluble in clarified whey.

Clarified whey can be used either for preparing crystal clear soft drinks with or without addition of flavours, or as a pastry base with a high foaming capacity. For example, a pasteurized mix for meringue making can be prepared. On the other hand, uses of the sediment will be large and diversified not only in traditional dairy products but also in dietetic foods because of its high content in phospholipids.

Key words

Clarification - Whey - Interaction - Calcium - Lipoproteins.

\section{INTRODUCTION}

Le lactosérum, produit dérivé de l'industrie fromagère et caséinière contient des protéines de très haute valeur nutritionnelle (Forsum, 1974), ayant d'excellentes propriétés fonctionnelles notamment en ce qui concerne la solubilité, l'aptitude à structurer de l'eau (soit sous forme native, soit après dénaturation thermique) et le pouvoir moussant (de Wit et de Boer, 1975; Kinsella, 1976). Ces protéines, qui ne représentent que 12 à $15 \%$ de la substance sèche du lactosérum, peuvent être concentrées et purifiées par 
divers procédés utilisant les caractéristiques biochimiques ou physico-chimiques de ces macromolécules ou des constituants du lactosérum auxquels elles sont associées. Parmi ces procédés, 1'ultrafiltration sur membrane est certainement celui qui connaît le développement le plus important puisque la surface estimée de membrane installée dans l'industrie laitière mondiale pour le traitement du lactosérum doit maintenant dépasser les $100000 \mathrm{~m}^{2}$, ce qui représente une capacité de traitement de $30 \times 10^{9} 1 /$ an. Les concentrés de protéines de lactosérum (CPL) obtenus par cette technologie trouvent de nombreux et vastes débouchés dans les industries d'aval que ce soit pour l'alimentation des jeunes animaux ou que ce soit en alimentation humaine (Maubois, 1982).

Mais aussi bien la mise en œuvre de la technique d'ultrafiltration que les débouchés des CPL se trouvent limités par la présence de lipides résiduels dans les lactosérum mis en œuvre. En effet, la présence de ces composants lipoprotéiques provenant pour l'essentiel de la membrane des globules gras du lait crée un frein au transfert d'eau au travers des membranes d'ultrafiltration par leurs propriétés hydrophobes (il peut également être supposé que les lipoprotéines du lactosérum sont un des constituants majeurs de la couche de polarisation), limite le taux de pureté des protéines dans les CPL à $80-90 \%$ et déprécie certaines de leurs propriétés fonctionnelles telles que le pouvoir moussant.

C'est probablement aussi dans la présence des lipoprotéines qu'il faut rechercher les difficultés de développement des technologies de séparation fine telles que la chromatographie sur échangeurs d'ions ou la gel-filtration. En effet, de par leurs propriétés amphiphiles et amphotères, ces lipoprotéines très souvent associées par des liaisons hydrophobes avec des protéines sériques sont irréversiblement fixées sur les sites réactifs et « empoisonnent » ainsi progressivement mais irrémédiablement les supports chromatographiques. C'est enfin l'accumulation des lipoprotéines dans le circuit réactionnel au-dessus de la membrane qui limite le fonctionnement des réacteurs enzymatiques de fragmentation des protéines du lactosérum.

Divers procédés d'élimination de la matière grasse résiduelle des lactosérums, donc de clarification de ces liquides, ont été proposés dans le but de solutionner ces difficultés de mise en œuvre des techniques de séparation des protéines de lactosérum et de valorisation des CPL obtenus. Dalan et coll. (1972), de Wit et de Boer (1975) ont constaté que la réduction de la force ionique accompagnée d'un ajustement du $\mathrm{pH}$ à 4,6 entraînait une agrégation des lipoprotéines qui pouvaient ensuite être séparées par décantation. La réduction de la force ionique était réalisée par déminéralisation à l'aide de résines échangeuses d'ions ou d'électrodialyseurs ou encore par diafiltration. Melachouris (1977) a proposé l'addition 
de polyphosphates à du lactosérum préalablement amené à pH 5,1, addition suivie d'une neutralisation à $\mathrm{pH} 7,0$. Dans le même ordre d'idées, Best et coll. (1982) ont préconisé l'utilisation d'agents polyanioniques tels que des dérivés de l'acide acrylique après ajustement du $\mathrm{pH}$ du lactosérum à une valeur comprise entre 4,0 et 5,0 . Ces technologies sont, certes, relativement aisées à mettre en œuvre mais leur coût (déminéralisation - décantation - emploi de polymères ou de phosphates) n'est pas toujours acceptable en regard des plus-values enregistrées au niveau de l'amélioration de performance dans la technique de séparation et/ou dans l'extension de valorisation des CPL. De plus, tous ces procédés conduisent à un CPL ayant un $\mathrm{pH}$ de 4,6 ce qui limite son utilisation ultérieure. Lee et Merson (1976), plus récemment Piot et coll. (1984) ont proposé l'emploi de la microfiltration sur membrane mais pour prometteurs que soient les résultats déjà obtenus avec cette technique, l'optimisation industrielle des équipements est encore loin d'être réalisée én raison de la difficulté de la séparation à réaliser.

Le procédé de clarification qui est proposé dans la présente étude est basé sur l'aptitude des lipoprotéines du lactosérum à s'agréger par l'intermédiaire des ions calcium sous l'action d'un traitement thermique. Les conditions optimales de clarification, à savoir celles conduisant à l'obtention d'un lactosérum de la limpidité la plus élevée possible avec le minimum de pertes en protéines ont été recherchées.

\section{MATERIEL ET METHODES}

\section{Lactosérum}

Les lactosérums utilisés dans les expérimentations étaient des lactosérums issus de fabrication mécanisée de fromages Emmental (Société Préval - 35360 Montauban de Bretagne) débourbés et écrémés par centrifugation classique à $48^{\circ} \mathrm{C}$. Les teneurs en matière grasse résiduelle après écrémage étaient de $0,2 \mathrm{~g} . \mathrm{l}^{-1}$.

Le calcium était ajouté dans le lactosérum sous forme de chlorure de calcium en solution à 50 p. $100(\mathrm{p} / \mathrm{v})$ dans de l'eau distillée. Le $\mathrm{CaCl}_{2}$ utilisé était dans les premières expérimentations du produit Merck $\left(\mathrm{CaCl}_{2}\right.$ pur, déshydraté, à grains fins) puis du $\mathrm{CaCl}_{2}$ technique, qualité alimentaire (Sté Langlois, Rennes), lorsque les volumes de lactosérum mis en œuvre atteignaient plusieurs centaines de litres. L'addition du chlorure de calcium entraînait une baisse de 0,2 unités du $\mathrm{pH}$ du lactosérum (se situant initialement entre 6,3 et 6,5 ). Trois valeurs du $\mathrm{pH}$ étaient étudiées :

- $\mathrm{pH}$ 6,6 ajusté avec de la soude concentrée (soude d : 1,38 Prolabo à $35 \%)$; 
$-\mathrm{pH}$ 6,0 ajusté avec une solution aqueuse à $50 \mathrm{~g} / \mathrm{p} 100(\mathrm{p} / \mathrm{v})$ d'acide citrique;

- $\mathrm{pH}$ 5,2 ajusté avec la même solution d'acide citrique.

\section{Traitements thermiques}

Les traitements thermiques étaient réalisés sur la plate-forme d'études du laboratoire comportant les éléments suivants :

- une cuve de 6001 (Goavec) à doubles parois, équipée d'un dispositif d'agitation;

- un bac de lancement;

- une pompe volumétrique Moineau dont le débit était régulé par un variateur électronique de fréquence couplé à un débitmètre électromagnétique Krohne;

- un échangeur à plaques Vicarb type $\mathrm{V}_{7}$;

- une chaudière électrique à production d'eau chaude; $\pm 0,1^{\circ} \mathrm{C}$

- un régulateur de température du fluide caloporteur à

- une réserve d'eau glacée avec stockage de glace.

Dans tous nos essais, le temps de chambrage était de 20 secondes et le débit de traitement de $300 \mathrm{l} / \mathrm{h}$. Chaque traitement thermique était réalisé sur des fractions aliquotes de 1001 de lactosérum.

\section{Décantation - Centrifugation - Filtration}

Après traitement thermique et refroidissement à $2^{\circ} \mathrm{C}$, une fraction du lactosérum était mise à décanter, pendant $24 \mathrm{~h}$, dans une éprouvette graduée d'un litre. Les hauteurs de sédiment étaient mesurées à intervalles réguliers. Une autre fraction était centrifugée pendant $30 \mathrm{~min}$ à $1000 \mathrm{~g}$ (centrifugeuse Mistral MSE 6 1). Le reste du lactosérum maintenu à $2^{\circ} \mathrm{C}$ était laissé à décanter dans le tank pendant $24 \mathrm{~h}$. Le surnageant était filtré sur équipement pilote Seitz Zenit V 40 équipé de plaques filtrantes Supra EK de porosité 1 micron (surface $1,10 \mathrm{~m}^{2}$ ).

\section{Déterminations analytiques}

La teneur en azote total était déterminée par la méthode Kjeldahl. Le coefficient 6,38 était utilisé pour la détermination des teneurs en matières azotées totales (MAT).

Les teneurs en $\beta$-lactoglobuline et en $\alpha$-lactalbumine étaient déterminées par chromatographie liquide haute pression (HPLC) avec une colonne TSK 3000 SW. Après injection de $10 \mu \mathrm{l}$ de lacto- 
sérum, l'élution était réalisée avec un tampon Tris $0,01 \mathrm{M}-\mathrm{HCl}$ $\mathrm{pH} 6,68$ au débit de $1 \mathrm{ml} / \mathrm{min}$ avec détection à $280 \mathrm{~nm}$.

Les teneurs en substance sèche étaient déterminées par dessication à l'étuve pendant $7 \mathrm{~h}$ à $102^{\circ} \mathrm{C}$.

Les teneurs en calcium étaient déterminées par absorption atomique selon Brulé et coll. (1974).

Les teneurs en phosphore étaient déterminées selon la méthode préconisée par la F.I.L. (norme 33).

Les $\mathrm{pH}$ étaient mesurés à l'aide d'un appareil Minisis 5000 Tacussel à 0,01 unité près.

Les teneurs en matière grasse des culots de centrifugation étaient déterminées par extraction à l'aide du mélange éther diéthylique-éther de pétrole (norme IDF $\mathrm{n}^{\circ} 5$ : 1959 de la F.I.L.).

La densité optique des surnageants de décantation ou de centrifugation était déterminée à l'aide d'un spectrophotomètre Beckman à $600 \mathrm{~nm}$ par rapport à un témoin constitué par de l'ultrafiltrat de lactosérum.

\section{RESULTATS}

\section{Influence de la température, de la dose de calcium ajouté et du pH sur la limpidité du lactosérum clarifié}

Le tableau 1 récapitule l'ensemble des observations réalisées sur les lactosérums centrifugés après traitement. A pH 5,2, il était constaté une très mauvaise clarification quelles que fussent les quantités de calcium ajouté et l'intensité du traitement thermique appliqué. La meilleure limpidité, appréciée visuellement, était obtenue lorsque le $\mathrm{pH}$ du lactosérum était égal ou supérieur à 6,0 , la teneur en calcium total était de l'ordre de 1,2 g.1 $1^{-1}$ ce qui correspondait à un ajout de 0,8 g.t $1^{-1}$ et lorsque le traitement thermique se situait entre 75 et $80^{\circ} \mathrm{C}$. Etant donné que le $\mathrm{pH}$ initial des lactosérums issus de la transformation du lait en fromage Emmental est, en général, voisin de 6,4-6,2 et que l'addition de $\mathrm{CaCl}_{2}$ à la dose de 2,22 g. $1^{-1}\left(0,8\right.$ g. $1^{-1}$ de $\left.C a\right)$ entraîne un abaissement de 0,2 unités $\mathrm{pH}$, l'ajustement à $\mathrm{pH} 6,0$ a été retenu pour la suite des expérimentations.

\section{Influence du traitement thermique, de la dose de calcium ajouté sur la composition du lactosérum clarifié}

La figure 1 montre qu'aux températures inférieures ou égales à $80^{\circ} \mathrm{C}$ et au $\mathrm{pH}$ d'ajustement retenu $(6,0)$, la teneur en MAT du lactosérum centrifugé après traitement était supérieure à $80 \%$ de celle du lactosérum initial quelle que fût la dose de $\mathrm{Ca}$ ajouté. La pré- 


\section{TABLEAU 1 - TABLE 1}

Influence de la température, du calcium ajouté et du $\mathrm{pH}$ sur la limpidité du lactosérum clarifié

Visual observations on influence of temperature, added calcium content and $\mathrm{pH}$ on clearness of clarified whey

\begin{tabular}{|c|c|c|c|c|c|c|c|c|c|c|c|c|}
\hline \multirow{2}{*}{$\begin{array}{c}\text { Température } \\
\text { du } \\
\text { traitement }\end{array}$} & \multicolumn{4}{|c|}{$\begin{array}{c}\mathrm{pH} 6,6 \\
\text { Calcium }\end{array}$} & \multicolumn{4}{|c|}{$\begin{array}{l}\mathrm{pH} 6,0 \\
\text { Calcium }\end{array}$} & \multicolumn{4}{|c|}{$\begin{array}{l}\mathrm{pH} \mathrm{5,2} \\
\text { Calcium }\end{array}$} \\
\hline & 0 & 0,39 & 0,57 & 0,76 & 0 & 0,36 & 0,61 & 0,74 & 0 & 0,42 & 0,46 & 0,86 \\
\hline $65^{\circ} \mathrm{C}$ & TT & TT & - & $\mathrm{T}$ & TT & TT & $\mathrm{TT}$ & $\mathrm{T}$ & TT & TT & $\mathrm{TT}$ & TT \\
\hline $70^{\circ} \mathrm{C}$ & $\mathrm{TT}$ & $\mathrm{TT}$ & - & ML & TT & TT & $\mathrm{T}$ & ML & $\mathrm{TT}$ & TT & $\mathrm{TT}$ & $\mathrm{TT}$ \\
\hline $75^{\circ} \mathrm{C}$ & $\mathrm{TT}$ & $\mathrm{T}$ & ML & L & $\mathrm{TT}$ & $\mathrm{T}$ & $\mathrm{T}$ & L & $\mathrm{TT}$ & $\mathrm{T}$ & $\mathrm{T}$ & $\mathrm{T}$ \\
\hline $80^{\circ} \mathrm{C}$ & $\mathrm{TT}$ & ML & $\mathrm{L}$ & TL & $\mathrm{TT}$ & ML & ML & TL & TT & $\mathrm{T}$ & $\mathrm{T}$ & $\mathrm{T}$ \\
\hline $85^{\circ} \mathrm{C}$ & $\mathrm{TT}$ & $\mathrm{T}$ & $\mathrm{T}$ & - & TT & $\mathrm{T}$ & $\mathrm{T}$ & $\mathrm{T}$ & $\mathrm{TT}$ & TT & $\mathrm{TT}$ & $\mathrm{TT}$ \\
\hline $\begin{array}{l}\text { Très trouble } \\
\text { Trouble } \\
\text { Moyennement }\end{array}$ & $\lim$ & ipide & $\begin{array}{ll}\vdots & \mathrm{T} \\
\vdots & \mathrm{T} \\
\vdots & \mathrm{N}\end{array}$ & & & $\begin{array}{l}\text { Limp } \\
\text { Très }\end{array}$ & $\begin{array}{l}\text { ide } \\
\text { lim }\end{array}$ & & $\begin{array}{l}\mathrm{L} \\
\mathrm{TL}\end{array}$ & & & \\
\hline
\end{tabular}

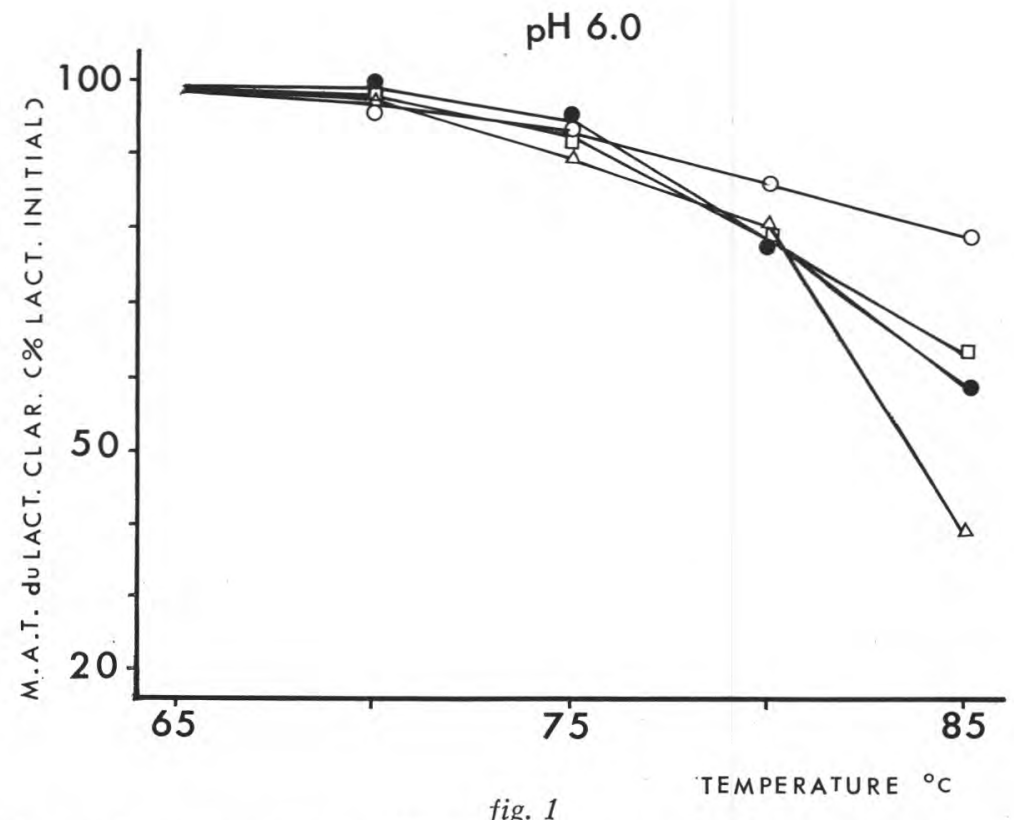

Evolution du pourcentage des MAT du lactosérum clarifié par rapport aux MAT du lactosérum initial en fonction de la température du traitement thermique et de la teneur en calcium ajouté $\left(\circ-\mathrm{O}\right.$ pas de $\mathrm{Ca}$ ajouté - $\square-\square \quad 0,36 \mathrm{~g} \cdot \mathrm{l}^{-1} \mathrm{de} \mathrm{Ca}$ ajouté - $-\bullet 0,61$ g.1-1 de Ca ajouté $-\triangle \longrightarrow \triangle 0,74$ g.1 $1^{-1}$ de Ca ajouté) à pH 6,0.

Evolution of total nitrogen in clarified whey ( $p$. cent) compared with total nitrogen of initial whey according to heat treatment temperature and amount of added calcium at $p H \quad 6.0$ ( $\mathrm{-} \bigcirc$ no added $\mathrm{Ca}-\square-\square \quad 0.36$ g.t-1 added $\mathrm{Ca}-\bullet-\bullet$ 0.61 g.l-1 added $\mathrm{Ca}-\triangle \longrightarrow \triangle 0.74$ g.l $\mathrm{l}^{-1}$ added $\left.\mathrm{Ca}\right)$. 
cipitation des protéines était accentuée, à la fois par l'élévation de la température du traitement et par un accroissement de l'ajout de $\mathrm{Ca}$. A $85^{\circ} \mathrm{C}$ et pour une addition de $\mathrm{Ca}$ égale à $0,74 \mathrm{~g} \cdot \mathrm{l}^{-1}$, le précipité représentait $64 \%$ des MAT.

Pour des températures de traitement inférieures ou égales à $78^{\circ} \mathrm{C}$, l'addition de calcium n'avait que peu d'influence sur la proportion de $\beta$-lactoglobuline dénaturée par le traitement appliqué au lactosérum (fig. 2). Il restait dans le surnageant environ $85 \%$ de la $\beta$-lactoglobuline initiale. Par contre, à $85^{\circ} \mathrm{C}$ quelle que fût la dose de $\mathrm{Ca}$ ajouté, le taux de dénaturation de cette protéine atteignait $78 \%$. Cette dénaturation se réduisait à $32 \%$ lorsqu'il n'était pas ajouté de $\mathrm{Ca}$ au lactosérum avant traitement thermique. Le taux de dénaturation de l'a-lactalbumine restait toujours inférieur à $20 \%$ quelles que fussent la température de traitement et la dose de $\mathrm{Ca}$ ajouté (fig. 3).

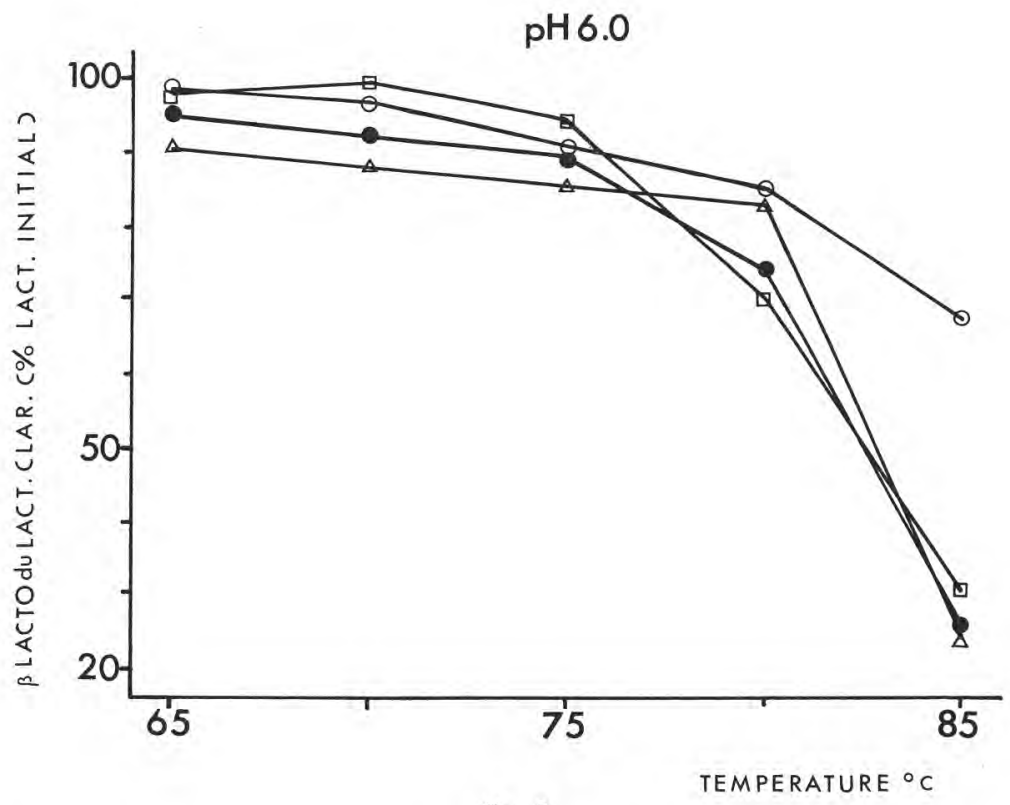

fig. 2

Evolution du pourcentage de la $\beta$ lactoglobuline du lactosérum clarifié par rapport à la $\beta$ lactoglobuline du lactosérum initial en fonction de la température du traitement thermique et de la teneur en calcium ajouté ( $0-0$ pas de $\mathrm{Ca}$

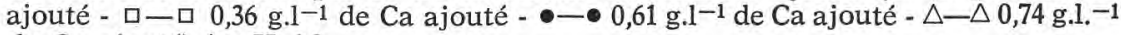
de $\mathrm{Ca}$ ajouté) à $\mathrm{pH}$ 6,0.

Evolution of $\beta$-lacto content in clarified whey ( $p$. cent) compared with $\beta$-lacto content of initial whey according to heat treatment temperature and amount of added calcium at $\mathrm{pH} 6.0$ ( $\mathrm{O}-\mathrm{O}$ no added $\mathrm{Ca}-\mathrm{\square}-\mathrm{\square} 0.36 \mathrm{~g} . \mathrm{l}^{-1}$ added $\mathrm{Ca}-\bullet-0.61$ g. $l^{-1}$ added $\mathrm{Ca}-\triangle-\triangle 0.74$ g. $\mathrm{l}^{-1}$ added $\left.\mathrm{Ca}\right)$. 


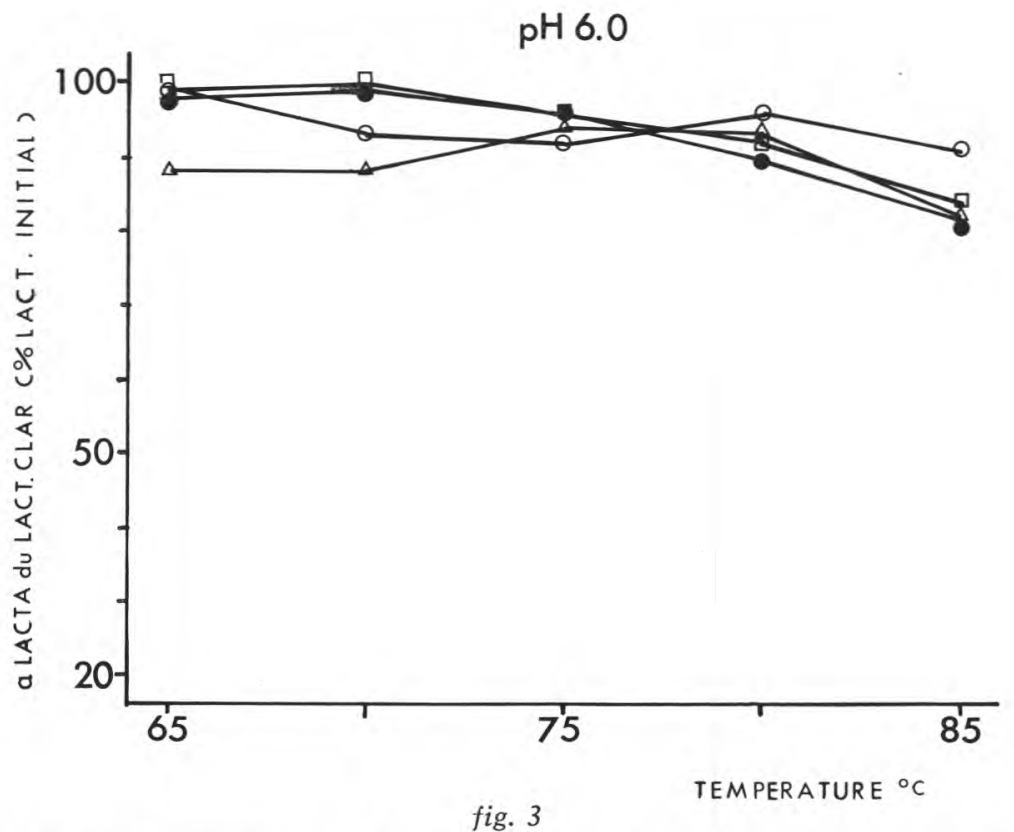

Evolution du pourcentage de l' $\alpha$ lactalbumine du lactosérum clarifié par rapport à l' $\alpha$ lactalbumine du lactosérum initial en fonction de la température du traitement thermique et de la teneur en calcium ajouté (O- $\mathrm{O}$ pas de $\mathrm{Ca}$ ajouté -

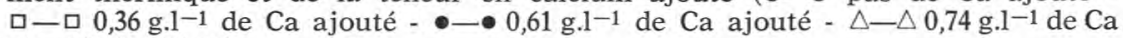
ajouté) à $\mathrm{pH} 6,0$.

Evolution of $\alpha$-lacta content in clarified whey ( $p$. cent) compared with $\alpha$-lacta content of initial whey according to heat treatment temperature and amount of added calcium at pH 6.0 (O-० no added $\mathrm{Ca}-\square-\square 0.36$ g.t-1 added $\mathrm{Ca}$ $\bullet-0.61$ g.l-1 added $\mathrm{Ca}-\triangle-\triangle 0.74$ g.l added $\mathrm{Ca}$ ).

Les figures 4 et 5 montrent qu'au $\mathrm{pH}$ d'ajustement retenu, les teneurs en calcium et en phosphore des surnageants de lactosérum étaient peu modifiées par le traitement thermique quelle que fût la dose de calcium ajouté.

\section{Optimisation des paramètres de clarification}

Dans le but de trouver le meilleur compromis possible entre une clarification maximale du lactosérum et une dénaturation minimale des protéines, une étude systématique de l'influence de la température de traitement thermique entre $70^{\circ}$ et $83^{\circ} \mathrm{C}$ a été entreprise sur les proportions de MAT, de $\beta$-lactoglobuline et d' $\alpha$-lactalbumine restant dans les surnageants de lactosérum (fig. 6), le pH d'ajustement du lactosérum restant fixé à 6,0 et la dose de calcium ajouté à 0,8 g. $\mathrm{l}^{-1}$. La proportion de MAT restant dans le lactosérum 


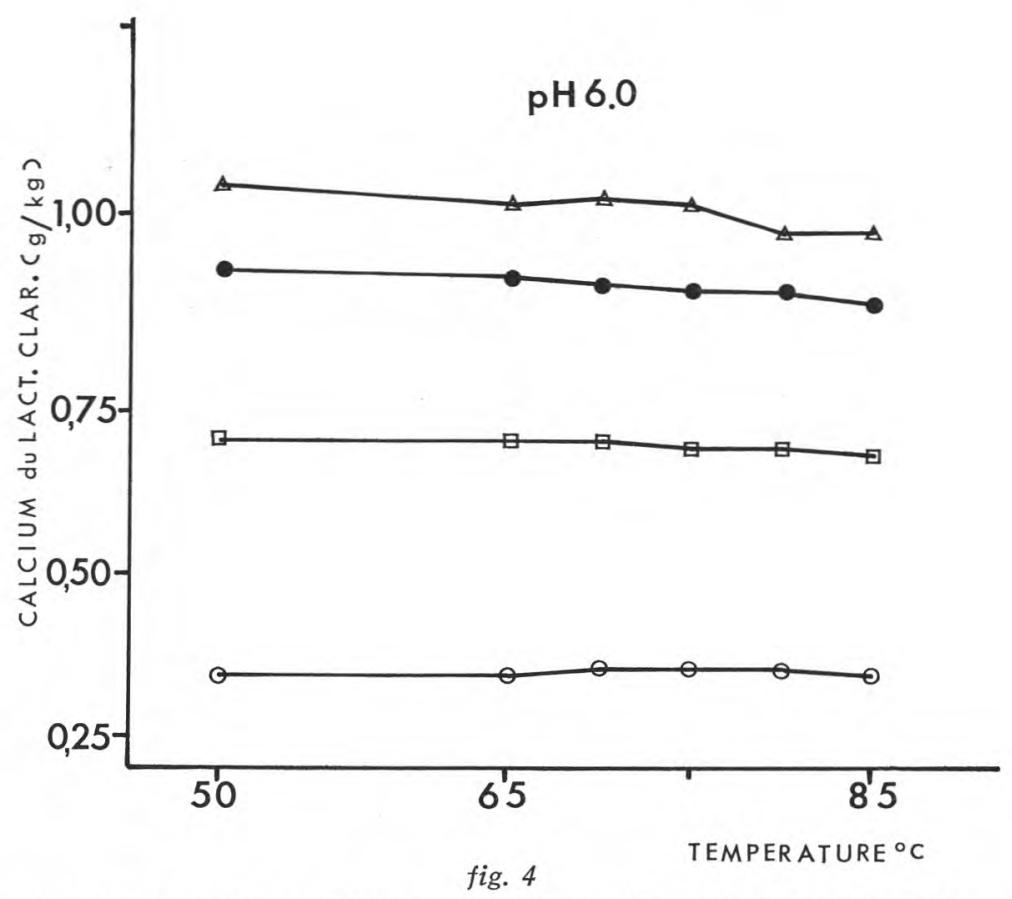

Evolution de la teneur en calcium dans le lactosérum clarifié en fonction de la température du traitement thermique et de la teneur en calcium ajouté $(0-0$ pas de calcium ajouté - ㄴ 0,36 g.1 ${ }^{-1}$ de calcium ajouté $-\bullet-\bullet 0,61$ g.1 $1^{-1}$ de calcium ajouté - $\triangle-\triangle 0,74 \mathrm{~g} \cdot \mathrm{1}^{-1}$ de $\mathrm{Ca}$ ajouté) à $\mathrm{pH} 6,0$.

Evolution of calcium content in clarified whey according to heat treatment temperature and amount of added calcium at $p H 6.0$ (O-O no added $\mathrm{Ca}$ ローロ 0.36 g.l-1 added $\mathrm{Ca}-\bullet \longrightarrow 0.61$ g.t $\mathrm{l}^{-1}$ added $\mathrm{Ca}-\triangle \longrightarrow \triangle 0.74$ g.l-1 added $\mathrm{Ca}$ ).

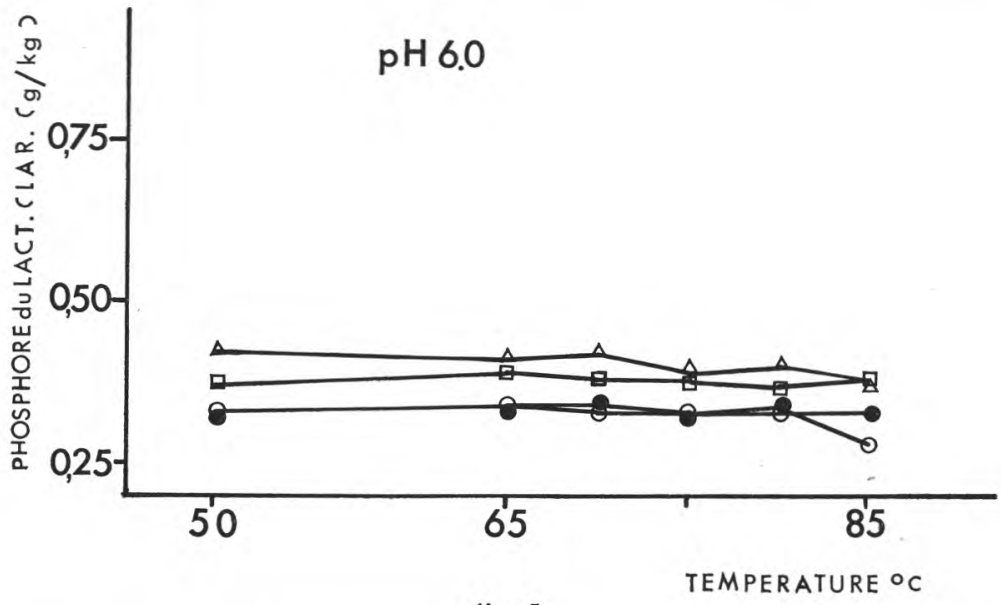

fig. 5

Evolution de la teneur en phosphore dans le lactosérum clarifié en fonction de la température du traitement thermique et de la teneur en calcium ajouté

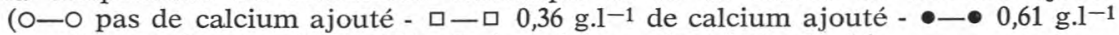
de calcium ajouté - $\triangle-\triangle 0,74 \mathrm{~g} / \mathrm{l}$ de calcium ajouté) à $\mathrm{pH}$ 6,0.

Evolution of phosphorus content in clarified whey according to heat treatment temperature and amount of added calcium at $\mathrm{pH} 6.0$ (O-O no added $\mathrm{Ca}$ -

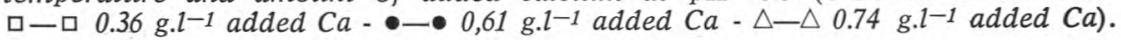




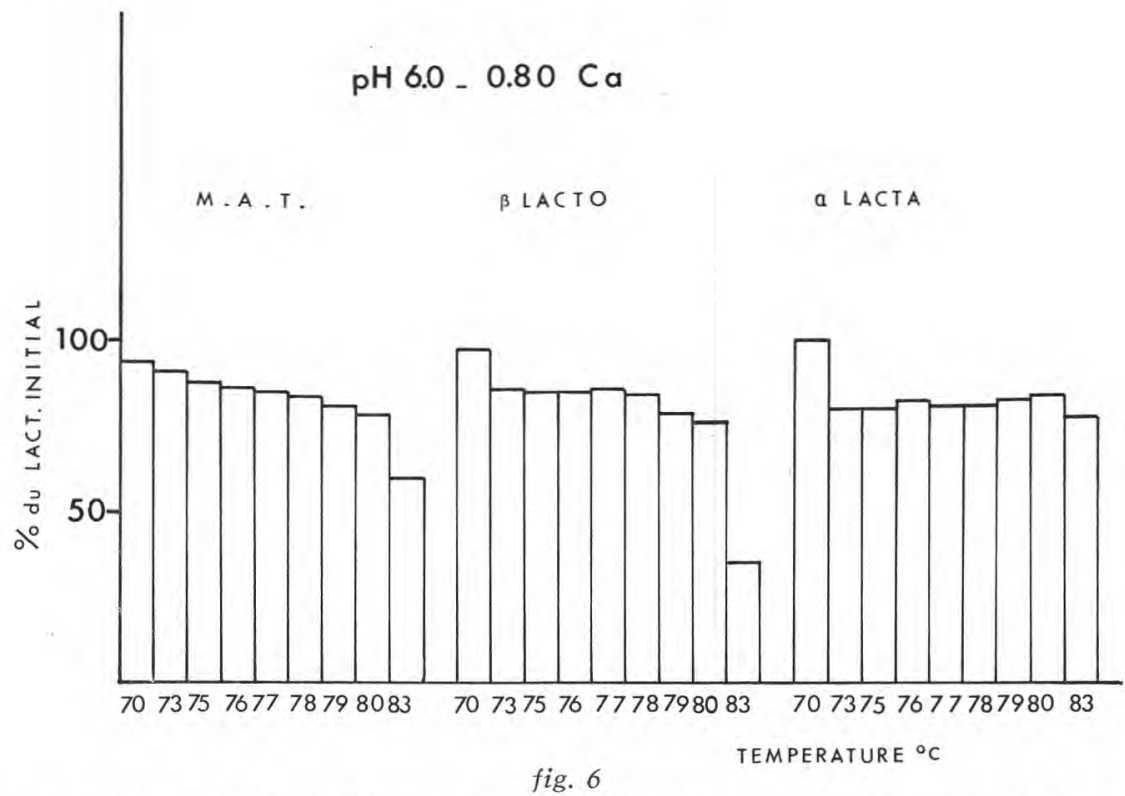

Proportion de MAT, de $\beta$ lactoglobuline et d' $\alpha$ lactalbumine restant dans le lactosérum clarifié en fonction de la température du traitement thermique au $\mathrm{pH}$ 6,0 et à la teneur de $0,80 \mathrm{~g} . \mathrm{l}^{-1}$ de calcium ajouté.

Proportion of total nitrogen, $\beta$-lacto and $\alpha$-lacta remaining in clarified whey according to heat treatment temperature of whey at $p H 6.0$ and with 0.8 g.l-1 calcium added.

clarifié diminuait légèrement mais régulièrement avec l'accroissement de la température du traitement; elle représentait $88 \%$ à $75^{\circ} \mathrm{C}, 84 \%$ à $78^{\circ} \mathrm{C}, 81 \%$ à $79^{\circ} \mathrm{C}$. Cette diminution des MAT était principalement due à une dénaturation de la $\beta$-lactoglobuline qui atteignait respectivement pour les mêmes températures 85,84 et $79 \%$, la proportion d' $\alpha$-lactalbumine présente dans le surnageant restant voisine de $82 \%$ de la teneur en $\alpha$-lactalbumine du lactosérum initial. La température de $80^{\circ} \mathrm{C}$ apparaît comme une valeur seuil au delà de laquelle il se produisait une chute brutale de la teneur en MAT du lactosérum clarifié due à une importante dénaturation de $\beta$-lactoglobuline.

Une appréciation objective de la limpidité des lactosérums clarifiés centrifugés était réalisée, dans cette série d'expérimentations, par la mesure de la densité optique (DO) à $600 \mathrm{~nm}$ (fig. 7). Le maximum de limpidité correspondant à une $\mathrm{DO}$ minimale était obtenu par un traitement thermique à $80^{\circ} \mathrm{C}$. Le lactosérum clarifié résultant du traitement à cette température a une limpidité proche de celle d'un ultrafiltrat de lait ou de lactosérum (DO supérieure de 0,01 unité). 
TABLEAU $2-T A B L E 2$

Caractéristiques et résultats de la filtration sur filtre Seitz - Characteristics and results of filtration on Seitz filter

\begin{tabular}{|c|c|c|c|c|c|}
\hline & $\begin{array}{c}\text { Surnageant } \\
\text { avant filtration }\end{array}$ & $\begin{array}{l}\text { Début } \\
\text { filtration }\end{array}$ & $\begin{array}{l}\text { Après } 15 \mathrm{~min} \\
\text { de filtration }\end{array}$ & $\begin{array}{l}\text { Après } 30 \mathrm{~min} \\
\text { de filtration }\end{array}$ & $\begin{array}{l}\text { Après } 60 \mathrm{~min} \\
\text { de filtration }\end{array}$ \\
\hline $\begin{array}{l}\text { Densité } \\
\text { optique }\end{array}$ & 0,062 & 0,004 & 0,007 & 0,011 & 0,009 \\
\hline $\begin{array}{l}\text { Débit de } \\
\text { filtration } \\
1 . h^{-1} \mathrm{~m}^{-2}\end{array}$ & 380 & 380 & 380 & 380 & 380 \\
\hline $\begin{array}{l}\text { Pression } \\
\text { à l'entrée } \\
\text { bar }\end{array}$ & & 0,4 & 0,4 & 0,4 & 0,5 \\
\hline Aspect & trouble & incolore & incolore & $\begin{array}{c}\text { coloré jaune } \\
\text { verdâttre }\end{array}$ & $\begin{array}{l}\text { coloré jaune } \\
\text { verdấtre }\end{array}$ \\
\hline
\end{tabular}




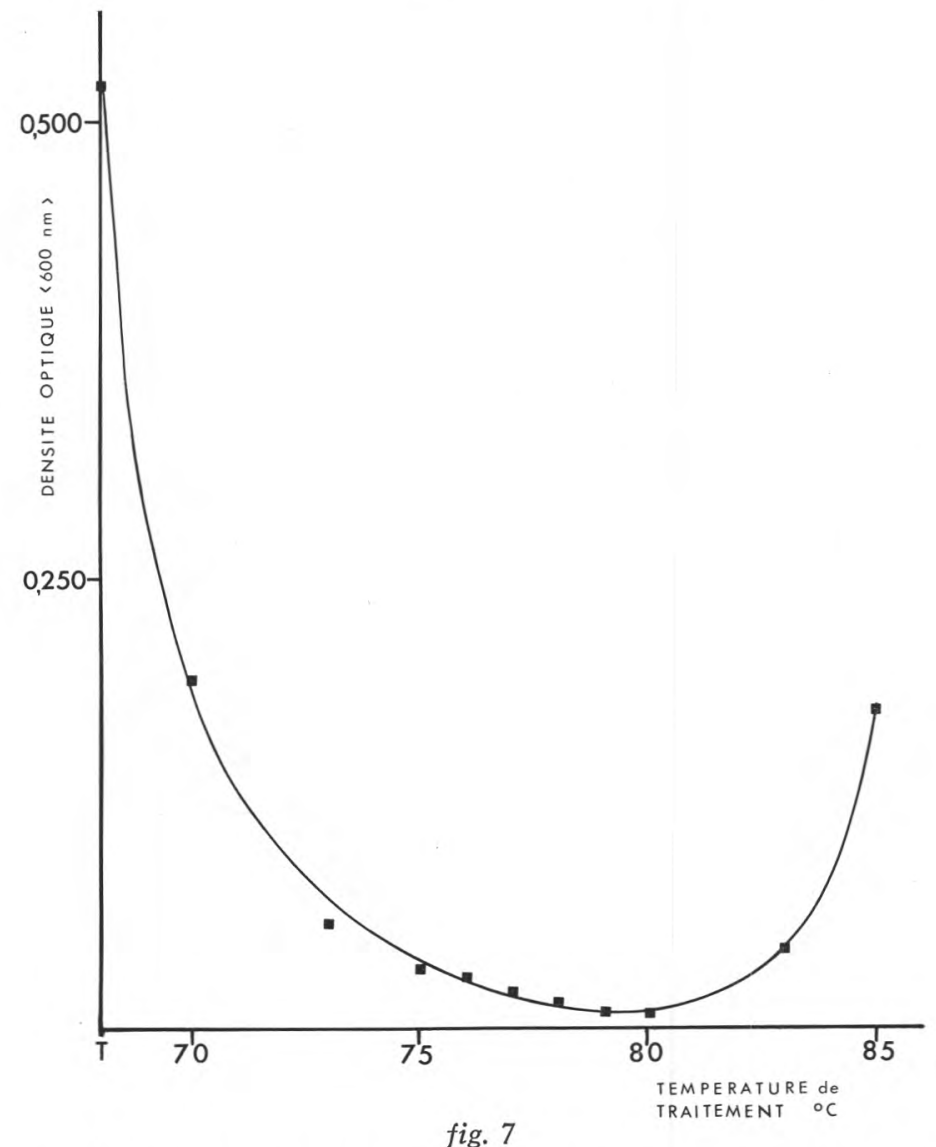

Evolution de la densité optique à $600 \mathrm{~nm}$ des surnageants des lactosérums clarifiés.

Evolution of optical density at $600 \mathrm{~nm}$ in clarified whey supernatants.

La prise en compte des résultats rassemblés dans les figures 6 et 7 permet de dégager les paramètres optima de clarification, à savoir ajustement du $\mathrm{pH}$ du lactosérum à 6,0, addition de $\mathrm{Ca}$ à la dose de $0,8 \mathrm{~g} .1^{-1}$ et traitement thermique à une température de $78-79^{\circ} \mathrm{C}$. Dans ces conditions, la dénaturation protéique était limitée à $17 \%$ des MAT du lactosérum initial et la DO était minimale.

\section{Cinétique de décantation da précipité - Filtration du surnageant}

Les résultats obtenus lors de l'étude de la cinétique de décantation d'un lactosérum traité dans les conditions optimales définies ci-dessus et refroidi à $2^{\circ} \mathrm{C}$ sont rassemblés dans la figure 8 . Au 


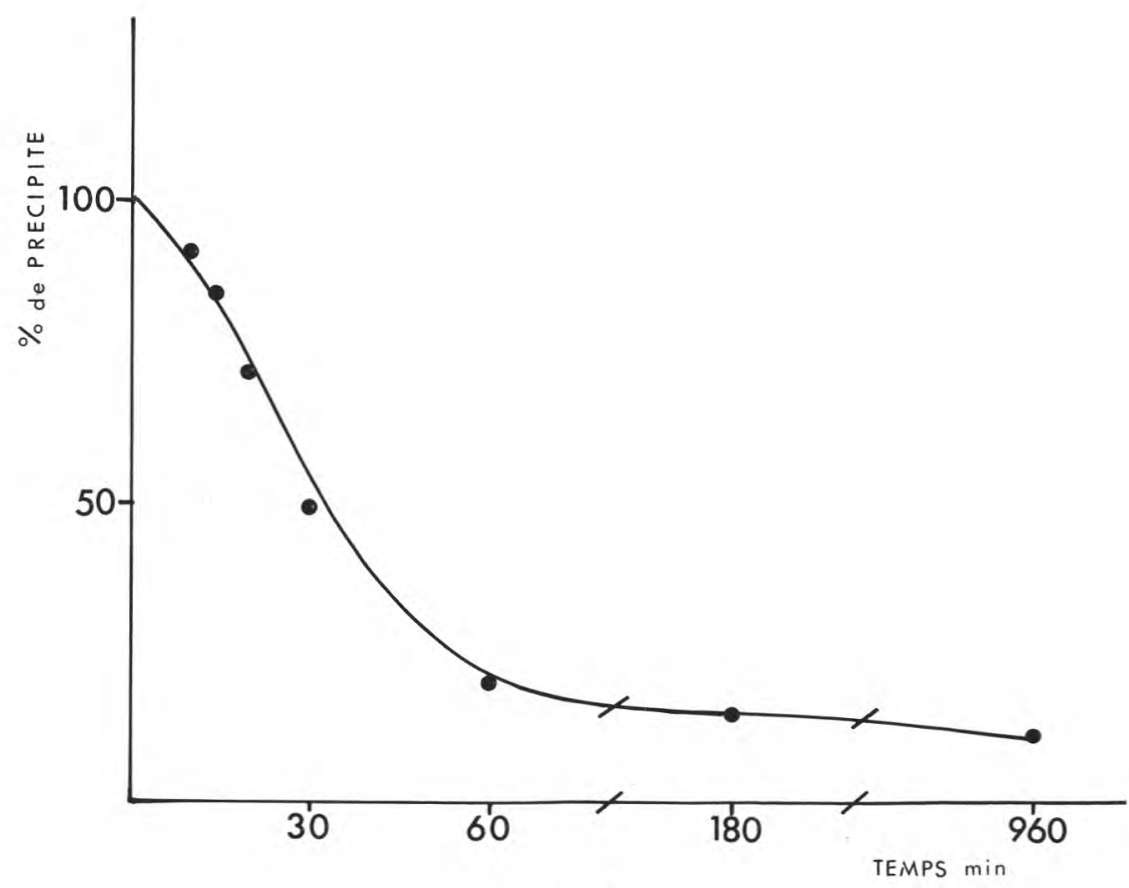

fig. 8

Cinétique de décantation du précipité à $2^{\circ} \mathrm{C}$.

Kinetics of precipitate decanting at $2^{\circ} \mathrm{C}$.

bout de $30 \mathrm{~min}$ de repos, le décantat occupait $50 \%$ du volume de l'éprouvette. A $60 \mathrm{~min}$, il ne représentait qu'à peine $25 \%$ du volume total mais il fallait attendre $16 \mathrm{~h}$ pour atteindre le niveau minimal de $10 \%$.

Les surnageants de décantation des lactosérums ainsi obtenus n'avaient pas une limpidité équivalente à celle des surnageants de centrifugation issus des mêmes lactosérums. Leurs DO respectives étaient de 0,06 et de 0,01. Les essais de mise en œuvre industrielle de clarification de ces surnageants de décantation à l'aide de séparateurs centrifuges et de bactofuges utilisés dans les usines laitières s'étant révélés infructueux, des essais de filtration en flux perpendiculaire sur filtre presse classique ont été réalisés à l'aide du matériel disponible au laboratoire. Les caractéristiques de filtration et les résultats obtenus sont indiqués dans le tableau 2. Les valeurs de DO inférieures à 0,01 constatées sur les 100 premiers litres filtrés étaient certainement dues à une adsorption de la riboflavine par les plaques filtrantes. En effet, il était constaté que les premières fractions de filtrat étaient totalement incolores et qu'une 
TABLEAU 3 - TABLE 3

Caractéristiques des produits obtenus après une thermisation à $79^{\circ} \mathrm{C}$ pendant $20 \mathrm{~s}$ d'un lactosérum additionné de $0,8 \mathrm{~g} \cdot 1^{-1}$ de $\mathrm{Ca}$ et dont le $\mathrm{pH}$ a été ajusté à 6,0

Characteristics of products obtained from whey with 0.8 g.l . $^{-1}$ Ca added ( $p H$ ajusted to 6.0) after thermization

\begin{tabular}{|c|c|c|c|c|c|c|c|c|c|c|c|c|c|}
\hline & 䍃 & $\stackrel{\circ}{\circ}$ & 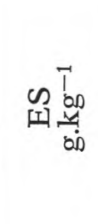 & 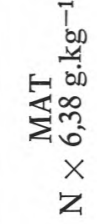 & 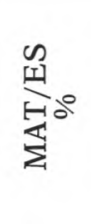 & 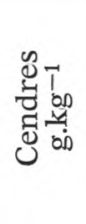 & 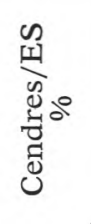 & 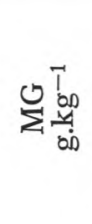 & $\sum_{\substack{\infty \\
0}}^{\infty}$ & 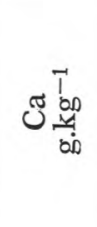 & م, & 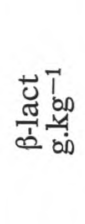 & 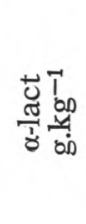 \\
\hline $\begin{array}{l}\text { Lactosérum } \\
\text { départ }\end{array}$ & 6,54 & 0,519 & 60,74 & 8,38 & 13,8 & 5,04 & 8,3 & 0,2 & & 0,323 & 0,411 & 4,37 & 1,37 \\
\hline $\begin{array}{l}\text { Lactosérum } \\
\text { + calcium }\end{array}$ & 6,00 & 0,522 & $63 ; 0$ & 8,36 & 13,27 & 7,03 & 11,15 & 0,2 & & 1,119 & 0,406 & 4,35 & 1,34 \\
\hline Surnageant & 5,93 & 0,012 & 60,97 & 6,99 & 11,46 & 6,84 & 11,22 & & & 1,042 & 0,332 & 3,46 & 1,17 \\
\hline Culot & & & 109,9 & 43,5 & 39,6 & 9,88 & 9,0 & 10,22 & 9,30 & 2,283 & 1,14 & & \\
\hline
\end{tabular}


coloration jaune-vert caractéristique de la présence de vitamine B 2 apparaissait progressivement après $15 \mathrm{~min}$ de filtration.

\section{Composition du décantat}

Les caractéristiques chimiques et physico-chimiques des produits obtenus au cours des différentes phases de la clarification d'un lactosérum dans les conditions optimales définies ci-dessus sont présentées dans le tableau 3. Le culot de centrifugation présentait une teneur en matière sèche égale à 1,7 fois celle du lactosérum additionné de $\mathrm{CaCl}_{2}$, sa teneur en MAT était 5,2 fois plus élevée, la matière grasse extractible était 51 fois celle du lactosérum. L'enrichissement en cendres n'était que de 1,4 fois, principalement en raison d'une coprécipitation de sels phosphocalciques.

\section{DISCUSSION}

La clarification du lactosérum doux de fromagerie est réalisable par agrégation des lipoprotéines qui y sont contenues, en présence de calcium ionisé et sous l'effet d'un traitement thermique. Cette fraction, responsable de l'opalescence, comprend à la fois des globules gras de très petite taille, non centrifugeables par les techniques classiques, et des fragments membranaires riches en phospholipides (sphingomyéline, phosphatidyléthanolamine, phosphatidylsérine, phosphatidylcholine) (Pitot, 1984) qui lui confèrent une très grande affinité vis-à-vis du calcium. Pour une valeur de $\mathrm{pH}$ comprise entre 6,6 et 6,0, l'addition de calcium ionique à ce type de lactosérum doit modifier les équilibres existant entre les formes ionisée et fixée de ce minéral, avec en particulier, un déplacement de calcium sur la fraction lipoprotéique. Ce déplacement doit être, en outre, accentué par un traitement thermique concomitant. La mise en nuvvre de cette technologie entraîne vraisemblablement, une agrégation des lipoprotéines et du calcium dont l'hydrophilie se trouve ainsi réduite, ce qui favorise la séparation des agrégats par décantation, par centrifugation ou par filtration. Cette réduction d'hydrophilie peut également être obtenue par régression d'ionisation des sites phosphoriques au moyen d'un abaissement du $\mathrm{pH}$ et d'une diminution de la force ionique (Dalan et coll., 1972; de Wit et de Boer, 1975).

La figure 9 schématise le procédé de clarification du lactosérum doux que nous proposons. L'addition de 0,8 g. $1^{-1}$ de calcium sous forme de $\mathrm{CaCl}_{2}$ et l'application d'un traitement thermique de $78-79^{\circ} \mathrm{C}$ pendant 20 secondes conduisent à un lactosérum d'une limpidité très voisine de celle d'un ultrafiltrat de lait ou de lactosérum, et ayant des teneurs en protéines et en minéraux légèrement modifiées par rapport à celles du lactosérum initial. La perte en protéines est 


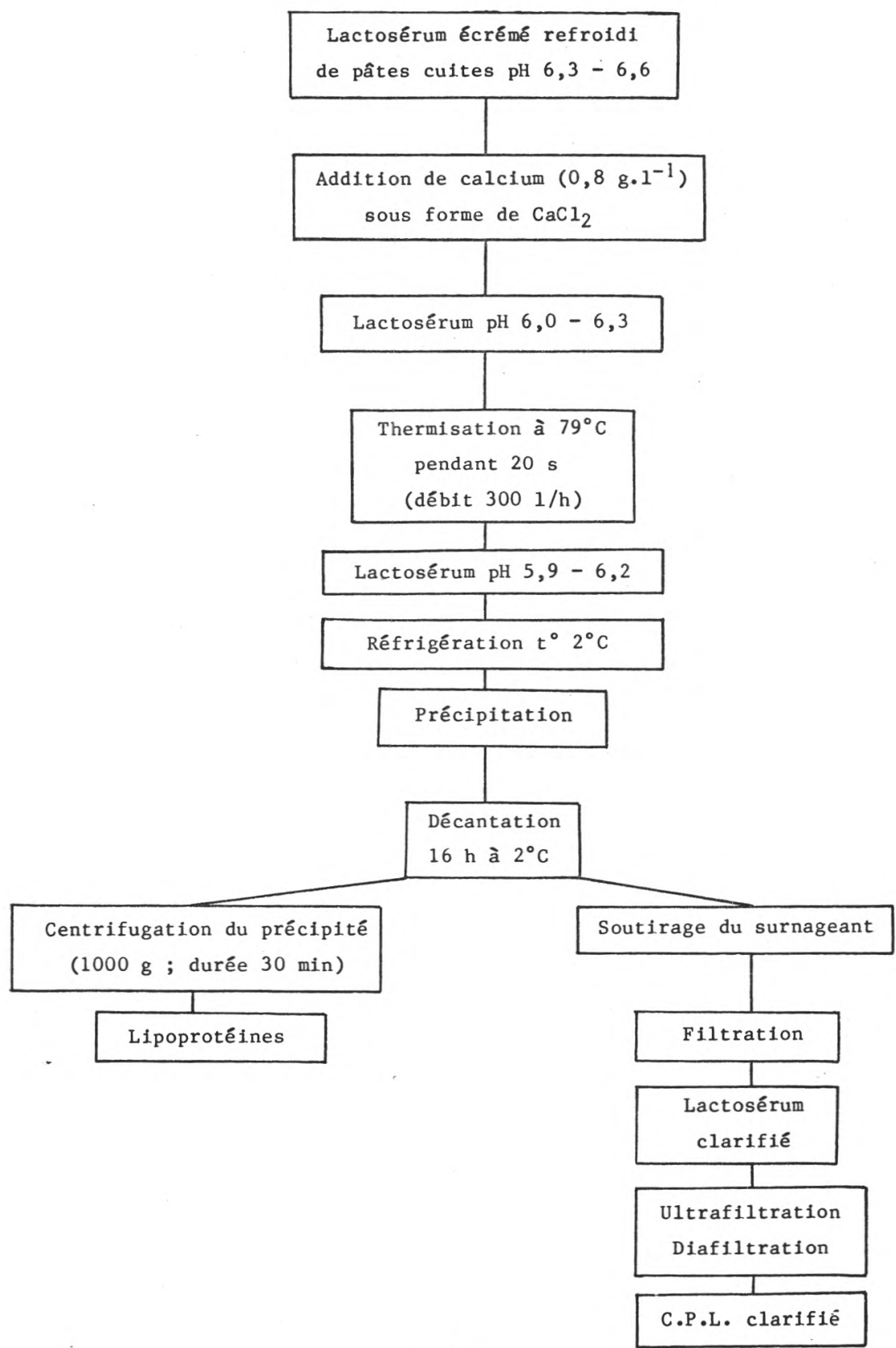

fig. 9

Procédé de clarification de lactosérum.

Whey clarification process. 
très dépendante de l'intensité du traitement thermique comme le montrent les résultats rassemblés dans la figure 6 . Une attention particulière devra donc être portée à la réalisation du chauffage du lactosérum additionné de $\mathrm{CaCl}_{2}$. Pour un temps de chambrage de 20 secondes, l'élévation à une température supérieure à $80^{\circ} \mathrm{C}$ entraîne une insolubilisation égale voire supérieure à $20 \%$ des protéines initiales, ce qui est inacceptable sur le plan technico-économique. Cette insolubilisation accrue résulte principalement d'une modification structurale de la $\beta$-lactoglobuline sous l'effet du traitement thermique, modification conduisant à un démasquage des sites susceptibles de chélater les ions calcium comme le montrent les résultats récents de de Wit (1984). Pour un même temps de chambrage de 20 secondes, une insolubilisation de l'ordre de $15 \%$ des protéines du lactosérum initial sera obtenue si la température atteinte est maintenue avec précision à $78,5^{\circ} \pm 0,5^{\circ} \mathrm{C}$, ce qui est tout à fait possible avec les équipements actuels de thermisation. Il devra, toutefois, être pris en considération, lors de l'éventuelle extrapolation industrielle du procédé que nous proposons, que les paramètres retenus pour le traitement thermique sont liés à l'équipement utilisé dans nos expérimentations. De légères adaptations peuvent s'avérer nécessaires avec l'utilisation d'autres équipements.

L'analyse électrophorétique montre que la $\beta$-lactoglobuline et l'a-lactalbumine représentent les $4 / 5$ des protéines insolubilisées, le reste étant constitué par la sérumalbumine bovine et les immunoglobulines (Fauquant, 1985). Ces dernières protéines, étant donné leur affinité vis-à-vis des constituants lipidiques (Stadhouders et Hup, 1970), jouent certainement un rôle déterminant dans la formation et l'agrégation des complexes lipoprotéiques, bien que ne représentant pas quantitativement une part importante des protéines du sédiment. Le calcium précipité ne représente que $7 \%$ du calcium alors que ce sont $17 \%$ du phosphore initial qui sont retrouvés dans le culot de centrifugation. Le rapport $\mathrm{Ca} / \mathrm{P}$ existant dans les agrégats qui décantent est voisin de 1 ce qui confirme la saturation par le calcium des résidus phosphoryl des lipoprotéines.

Du fait de sa limpidité, de l'élimination de son contenu lipidique, de sa bonne qualité bactériologique (moins de 500 germes $/ \mathrm{ml}$ ) et de son $\mathrm{pH}$, le lactosérum clarifié selon le procédé proposé constitue une nouvelle matière première particulièrement apte à la préparation de boissons aromatisées ou de substituts de blanc d'œuf. Des essais préliminaires ont montré qu'un rétentat d'ultrafiltration à $10 \mathrm{~g} \mathrm{p} 100$ de protéines, obtenu à partir de lactosérum clarifié avait un pouvoir moussant et une stabilité de la mousse formée tout à fait comparables à ceux observés avec du blanc d'œuf. De plus, additionné de $50 \mathrm{~g}$ p 100 de saccharose, ce rétentat pouvait être pasteurisé et constituer une base pâtissière de longue conservation. Sur un autre plan, il est fort probable que le fonc- 
tionnement et la durée de vie des colonnes de résines échangeuses d'ions utilisées pour la séparation fine des différentes protéines sériques seront grandement améliorées par la mise en œuvre de lactosérum clarifié, "l'empoisonnement » de ces résines par les lipoprotéines ne pouvant plus se réaliser. Les essais en cours confirment tout à fait cette conclusion.

Le complexe lipoprotéique séparé par décantation, par centrifugation ou par filtration du lactosérum a une composition tout à fait intéressante pour une valorisation ultérieure. Il présente en effet, des rapports protéines/matière sèche, matière grasse/matière sèche et protéines/matière grasse respectivement égaux à 0,$40 ; 0,09$ et 4,25. Sa richesse en phospholipides lui confère des propriétés émulsifiantes très élevées. De plus, ses caractéristiques nutritionnelles avec notamment un accroissement des teneurs en acides gras à chaîne moyenne $\left(\mathrm{C}_{10}\right.$ et $\left.\mathrm{C}_{14}\right)$ ainsi que celles en acides aminés (arginine et glycine) par rapport au lactosérum initial (Pitot, 1984) devraient lui ouvrir de larges débouchés aussi bien dans les produits laitiers classiques qu'en alimentation diététique.

\section{Remerciements}

Nous tenons à exprimer nos remerciements à l'équipe G.I.A. de l'I.N.R.A. de Rennes et à Mme Pierre pour l'aide et les précieux conseils qu'elles nous ont apportés au cours des essais de thermisation et dans la rédaction de cette publication.

\section{Bibliographie}

Best (E.), Plainer (H.), Sprossler (B.) (1982). - Method for removing substances causing turbidity in whey. European Patent Application EP 0057273 A2.

Brulé (G.), Maubois (J.L.), Fauquant (J.) (1974). - Etude de la teneur en minéraux des produits obtenus lors de l'ultrafiltration du lait sur membrane. Le Lait, 54, 600-615.

Daban (E.), Groux (M.J.A.), Hidalgo (J.) (1972). - Procédé de préparation d'une fraction soluble des protéines du petit-lait. Demande de brevet suisse, $\mathrm{n}^{\circ} 13272 / 72$.

DE WIT (J.N.), DE BoER (R.) (1975). - Procédé de clarification de liquides contenant des protéines du petit-lait. Demande de brevet néerlandais, $\mathrm{n}^{\circ} \quad 75 / 13645$.

DE WIT (J.N.) (1984). - Functional properties of whey proteins in food systems. Neth. Milk Dairy J., 38, 71-89.

Fauquant (J.), Pierre (A.) (1985). - Résultats non publiés.

FoRsum (R.) (1974). - Nutritional evaluation of whey protein concentrates and their fractions. $J$. Dairy Sci., 57 (6), 665-669.

KINSELLA (J.E.) (1976). - Functional properties of proteins in foods : a survey. Crit. Rev. Food Sci. Nutr., (4), 219-280.

LEE (D.N.), MERSon (R.L.) (1976). - Prefiltration of cottage cheese whey to reduce fouling of ultrafiltration membranes. J. Food Sci., 41, 403-410. 
Maubors (J.L.) (1982). - Les protéines du lactosérum extraites par ultrafiltration, dans : Protéines animales : extraits, concentrés et isolats en alimentation humaine. C.M. Bourgeois et P. Le Roux, éd. Apria-Lavoisier, $172-190$.

Melachouris (N.) (1977). - Process for the recovery of whey protein having improved solution clarity using polyphosphates. US Pat, 4, 043-990.

Piot (M.), Maubois (J.L.), Schaegis (P.), Veyre (R.), Luccioni (M.) (1984). - Microfiltration en flux tangentiel des lactosérums de fromagerie. Le Lait, 64, $102-120$.

Рiтот (P.) (1984). - Incorporation de lipides de sérum dans des crèmes de consommation : influence sur leurs propriétés fonctionnelles. Mémoire fin d'études E.N.S.F.A.

Stadhouders (J.), Hup (G.) (1970). - Complexity and specificity of euglobulin in relation to inhibition of bacteria and to cream rising. Neth. Milk Dairy J., 24, 79-95. 